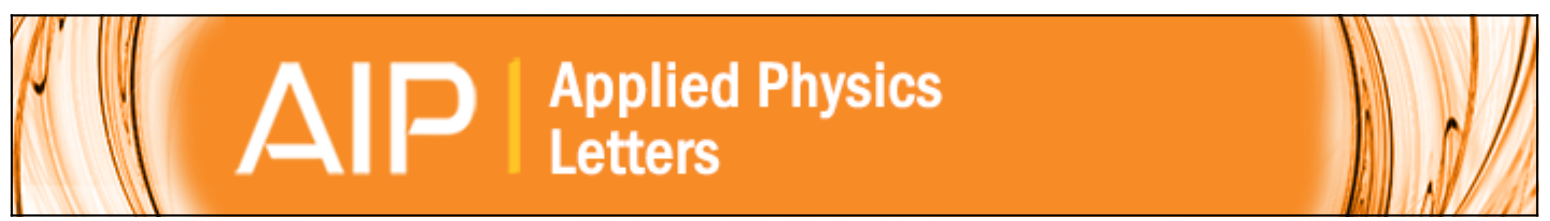

\title{
Surface charge dynamics on ferroelectric PbZr 0.48 Ti 0.5203 films responding to the switching bias of electric force microscope
}

J. Y. Son, Geunhee Lee, and Y.-H. Shin

Citation: Applied Physics Letters 94, 162902 (2009); doi: 10.1063/1.3124077

View online: http://dx.doi.org/10.1063/1.3124077

View Table of Contents: http://scitation.aip.org/content/aip/journal/apl/94/16?ver=pdfcov

Published by the AIP Publishing

\section{Articles you may be interested in}

Interface control of surface photochemical reactivity in ultrathin epitaxial ferroelectric films

Appl. Phys. Lett. 102, 182904 (2013); 10.1063/1.4802885

Electrical measurements of dielectric nonlinearities in ferroelectric bilayer thin films

J. Appl. Phys. 113, 074104 (2013); 10.1063/1.4792601

Photovoltaic characteristics in polycrystalline and epitaxial ( Pb 0.97 La 0.03 ) ( Zr 0.52 Ti 0.48 ) O 3

ferroelectric thin films sandwiched between different top and bottom electrodes

J. Appl. Phys. 105, 061624 (2009); 10.1063/1.3073822

Origin of surface potential change during ferroelectric switching in epitaxial PbTiO 3 thin films studied by scanning force microscopy

Appl. Phys. Lett. 94, 032907 (2009); 10.1063/1.3046786

Surface potential of ferroelectric thin films investigated by scanning probe microscopy

J. Vac. Sci. Technol. B 17, 1930 (1999); 10.1116/1.590851

Want to publish your paper in the \#1 MOST CITED journal in applied physics?

With Applied Physics Letters, you can.

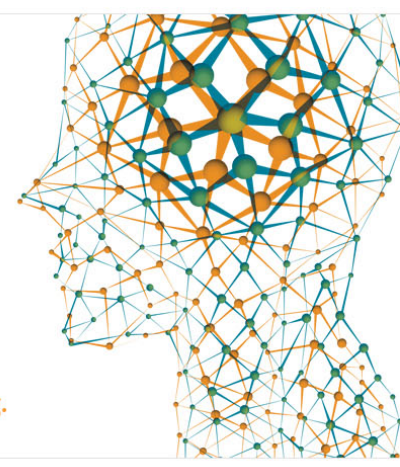




\title{
Surface charge dynamics on ferroelectric $\mathrm{PbZr}_{0.48} \mathrm{Ti}_{0.52} \mathrm{O}_{3}$ films responding to the switching bias of electric force microscope
}

\author{
J. Y. Son, Geunhee Lee, and Y.-H. Shin ${ }^{\text {a) }}$ \\ Department of Materials Science and Engineering, Pohang University of Science and Technology \\ (POSTECH), Pohang 790-784, Republic of Korea
}

(Received 28 February 2009; accepted 3 April 2009; published online 21 April 2009)

\begin{abstract}
We investigated the role of surface charges in writing and reading ferroelectric bits on an epitaxial $\mathrm{PbZr}_{0.48} \mathrm{Ti}_{0.52} \mathrm{O}_{3}$ thin film by electric force microscopy (EFM). The sign of EFM surface potential was reversed within several hundred microseconds for $10 \mathrm{~V}$. For a negative bias voltage of $-10 \mathrm{~V}$, EFM surface potential was reversed in several milliseconds. The different time scales of the EFM surface potential reversals originate from the screening of the ferroelectric polarization charges by the surface charges which pass over two different Schottky barriers depending on the applied bias polarity. (C) 2009 American Institute of Physics. [DOI: 10.1063/1.3124077]
\end{abstract}

Recently, there have been reported many researches on ferroelectric domain dynamics by using scanning probe microscope (SPM) due to its ability to observe ferroelectric domains in nanoscale resolution. ${ }^{1-3}$ Various SPM techniques such as piezoresponse force microscope (PFM), electric force microscope (EFM), and Kelvin probe force microscope (KFM) enable to switch and read ferroelectric polarization bits by using the electrostatic interaction between the polarization charge in a ferroelectric thin film and the reference charge at an SPM tip. ${ }^{3-10}$ In order to reverse polarization in ferroelectric capacitors, we need to apply an external electric field which is high enough to surmount the activation energy barrier to form critical nuclei. ${ }^{11,12}$ The external bias voltage should be applied for a sufficient time to flip ferroelectric polarization completely. ${ }^{13}$ Ferroelectric domain wall speeds can be estimated by observing the variation in the ferroelectric bit size as a function of SPM writing time. ${ }^{12,14,15}$ From the ferroelectric domain wall speeds, we can evaluate the activation energy for creeping motion of ferroelectric domain walls. ${ }^{12,14,15}$

The polarization of a switched ferroelectric domain bit gives rise to surface potential, which can be measured by using EFM or KFM. However, it was reported that various factors, such as ambient gas absorbed by ferroelectric thin films and surface charges induced from an SPM tip had influence on the surface potential measurement. ${ }^{5,8,10}$ Particularly, it was accepted that high bias voltages from an SPM tip can generate charges on the surface of ferroelectric thin films. ${ }^{10,12}$ In this study, we demonstrate that the EFM observation of an epitaxial (001) $\mathrm{PbZr}_{0.48} \mathrm{Ti}_{0.52} \mathrm{O}_{3}$ (PZT) thin film is influenced by the surface charges injected from an SPM tip during the switching of ferroelectric polarizations especially with higher bias voltages and longer writing time. Surface potential of ferroelectric polarization bits observed by EFM was reversed in several hundred microseconds for a positive bias voltage or in several milliseconds for a negative bias voltage. The different time scales of surface potential reversals observed by EFM originate from the screening of ferroelectric polarization charges by surface charges which surpass two different Schottky barriers between the EFM tip

\footnotetext{
${ }^{\text {a) }}$ Author to whom correspondence should be addressed. Electronic mail: yhshin@ postech.ac.kr.
}

and the PZT film depending on the applied bias polarity.

We prepared an epitaxial (001) PZT thin film deposited on a (100) $\mathrm{SrRuO}_{3} /(100) \mathrm{SrTiO}_{3}$ substrate by a pulsed laser deposition method. The thickness of the PZT thin film was about $50 \mathrm{~nm}$ with a high remanent polarization of $56 \mu \mathrm{C} / \mathrm{cm}^{2}$. To switch the polarity of ferroelectric domains, we applied a series of bias voltages between the conducting SPM tip (gold-coated Si cantilever) and the $\mathrm{SrRuO}_{3}$ bottom electrode for various writing times. After switching ferroelectric domains, we simultaneously measured surface potential and topography near the switched ferroelectric polarization bits. We carried out all EFM experiments in a nitrogen atmosphere to remove the influence of any other ambient gas with charged ions on the surface potential measurement.

In order to switch polarization of a ferroelectric bit in a dot shape $(<50 \mathrm{~nm}$, diameter), we used two opposite bias voltages of $\pm 10 \mathrm{~V}$ for writing times that ranged from $100 \mathrm{~ns}$ to $2 \mathrm{~ms}$. After switching polarization of the ferroelectric bit, we observed the ferroelectric bit with EFM, as shown in Fig. 1. The size of the ferroelectric bit increased with writing time. For the positive bias voltage $(10 \mathrm{~V})$, positive surface potential was observed in EFM signals for writing times of $100 \mathrm{~ns}-0.1 \mathrm{~ms}$ [Fig. 1(a)]. The positive surface potential reflects that the EFM signals are obtained from the Coulomb force between the EFM tip and the polarization charge of the ferroelectric bit. However, as the writing time was longer than $0.1 \mathrm{~ms}$, the EFM signal was reversed from positive to negative. Similarly to the EFM signal of $10 \mathrm{~V}$, the sign of the

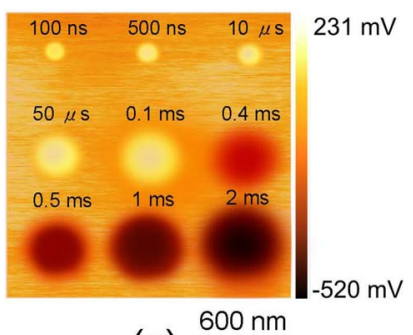

(a)

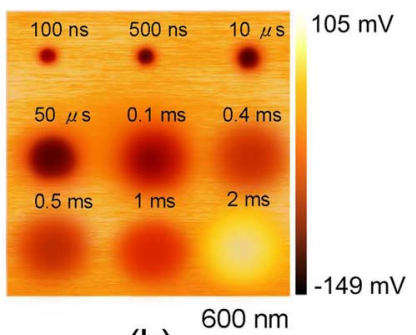

(b)
FIG. 1. (Color online) The EFM images of the PZT thin film after switching ferroelectric polarization bits with writing bias voltages of $\pm 10 \mathrm{~V}$. The reversal of the EFM signals was observed within (a) several microseconds for the writing bias voltage of $10 \mathrm{~V}$ and (b) several milliseconds for the writing bias voltage of $-10 \mathrm{~V}$. 


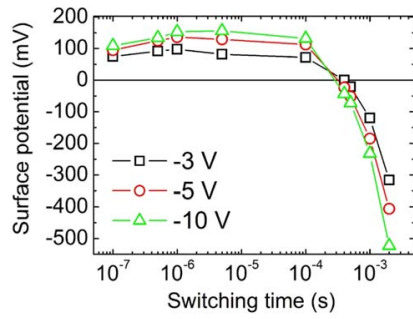

(a)

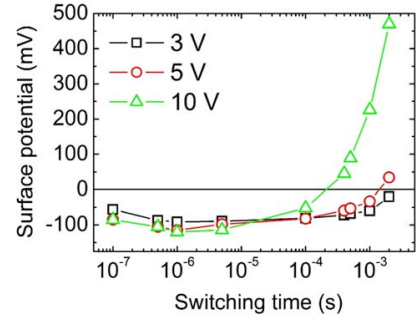

(b)
FIG. 2. (Color online) The surface potential as a function of writing time for the ferroelectric polarization bits with (a) negative $(-3,-5$, and $-10 \mathrm{~V})$ and (b) positive $(3,5$, and $10 \mathrm{~V})$ writing bias voltages.

EFM surface potential was changed from negative to positive after the external bias was applied for about $1 \mathrm{~ms}$ at -10 V [Fig. 1(b)].

Figure 2 shows surface potentials measured by EFM as a function of writing time for a series of bias voltages $( \pm 3$, \pm 5 , and $\pm 10 \mathrm{~V})$. Compared with the case of negative bias voltages, longer writing time was required to reverse the sign of the EFM signals for positive bias voltages. In particular, there is no reversal of surface potential for $+3 \mathrm{~V}$ until the writing time approaches to $2 \mathrm{~ms}$.

To understand the reversal of the EFM signals, we depicted a schematic drawing for switching of ferroelectric bits with EFM (Fig. 3). When the EFM tip is biased by positive or negative voltages, polarization charges at the PZT thin film and depolarization charges at the EFM tip are simultaneously formed on the ferroelectric surface, as shown in Fig. 3(a). When the bias voltage overcomes the Schottky barrier between the tip and the ferroelectric thin film, charges with the same polarity to the depolarization charges can be injected from the EFM tip to surface. ${ }^{16-18}$ These charges injected from the EFM tip (surface charges) are shown in Fig. 3(a). After the removal of the EFM tip from the surface of the PZT thin film, surface charges remained as depolarization charges on the surface of the PZT. ${ }^{13}$ Then, the surface charges are proportional to Schottky current and writing time, playing a role in screening the EFM signal of the ferroelectric polarization charges. ${ }^{10,13}$ As shown in Fig. 3(b), the reversal of surface potential can occur from the screening of the polarization charges by the surface charges. In the previous report, it was demonstrated that the surface charges can be discharged by a grounded EFM tip, indicating the evi-

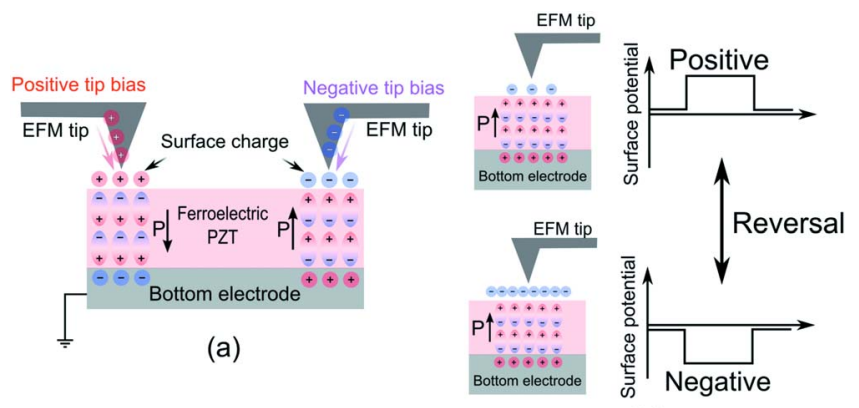

(b)

FIG. 3. (Color online) The schematic drawing of switching ferroelectric domains by an EFM tip. (a) Two oppositively polarized domains with different surface charges for positive and negative tip biases. (b) The EFM surface potential reversal by screening of the polarization charges by the surface charges (electrons for a negative tip bias)

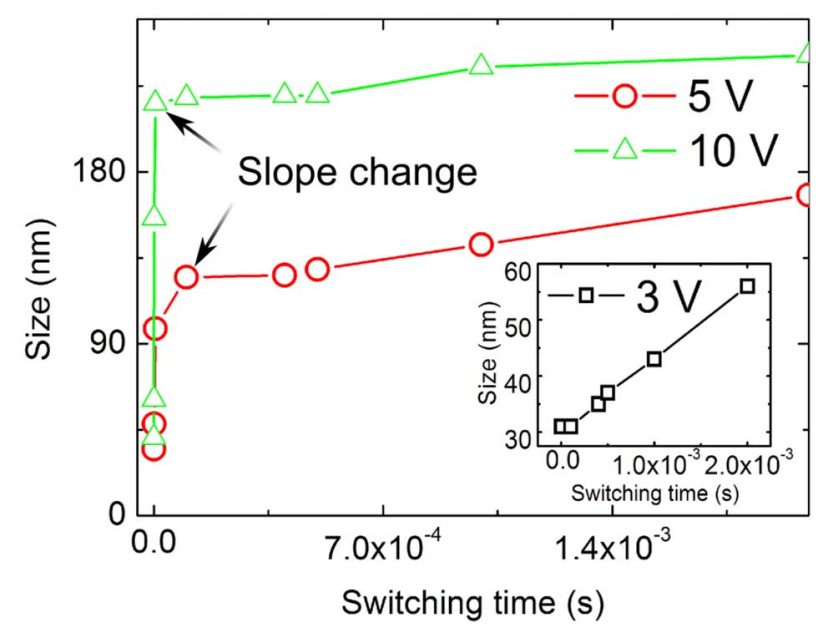

FIG. 4. (Color online) The size of the ferroelectric polarization bit as a function of writing time.

dence for the existence of surface charges. ${ }^{10}$ The comparative study of KFM and PFM on surface potential of $\mathrm{PbTiO}_{3}$ thin films also showed the role of surface charges in the observation of switched ferroelectric domains. ${ }^{19}$

The amount of the surface charges can be evaluated from the Schottky current $J=A T^{2} \exp \left(-q \Phi_{B} / k_{B} T\right)$ $\times \exp \left[1 / k_{B} T\left(q^{3} E / 4 \pi \varepsilon\right)^{1 / 2}\right]$, where $A$ is the Richardson constant, $q \Phi_{B}$ is the energy barrier to access the empty conduction-band states of the PZT thin film from the gold tip, $E$ is the applied electric field, and $\varepsilon$ is the dielectric constant of PZT. ${ }^{20}$ The electric field between the EFM tip and the surface can be written as $E=-(Q / 2 \pi \varepsilon) \int_{0}^{\infty} k J_{0}(k r)$ $\times[\exp (-k z)+\exp (k z-2 d-2 a)] /[1+\exp (-2 k d)] d k, \quad$ where $J_{0}(k r)$ is the zeroth-order Bessel function, $d$ is the thickness of the film, and $a$ is the tip radius. ${ }^{21}$ We estimate an electric field of about $0.48 \mathrm{MV} / \mathrm{cm}$ at the surface and about 0.16 $\mathrm{MV} / \mathrm{cm}$ at the bottom electrode for a bias voltage of $0.5 \mathrm{~V}$, where $d$ is $50 \mathrm{~nm}$ and $a$ is $30 \mathrm{~nm} .^{10,21,22}$ For $-3 \mathrm{~V}$, we obtain a Schottky current density of $80 \mathrm{~mA} / \mathrm{cm}^{2}$ with an electric field of $-2.86 \mathrm{MV} / \mathrm{cm}$. From this current density, we estimate a switching time of $0.70 \mathrm{~ms}$ at which the polarization charges of the PZT thin film are compensated. Thus, to inject surface charges enough to screen polarization charges, we need sufficient writing time more than several hundred microseconds for $-3 \mathrm{~V}$. However, for positive writing bias voltages, there exists a Schottky barrier of $2.5 \mathrm{eV}$, which is higher than that of $0.9 \mathrm{eV}$ for negative bias voltages because electrons flow from the surface of the ferroelectric thin film to the EFM tip under positive writing bias voltages. Thus, positive writing bias voltages require longer writing time to reverse the sign of the EFM signal. Therefore, we conclude that the different time scales of the EFM signal reversals originate from the screening of ferroelectric polarization charges by opposite charges which pass over two different Schottky barriers from the SPM tip to the PZT surface.

Figure 4 shows the diameter of a ferroelectric bit as a function of writing time. For 5 and $10 \mathrm{~V}$, each slope is clearly divided into two: initially the ferroelectric bit grows fast due to polarization flipping and surface charge dispersion, and then it grows slowly only through surface charge dispersion. The slope change occurs at the writing time when the EFM surface potential changes its sign For $3 \mathrm{~V}$, the bit size grows 
slowly because there is no reversal of the ferroelectric polarization bit.

In conclusion, we demonstrated the effect of the surface charges during writing and reading of ferroelectric polarization bits on the epitaxial PZT thin film with EFM. The different time scales of the surface potential reversal result from the screening of the polarization charges by the surface charges injected across the Schottky barrier between the EFM tip and the PZT thin film.

This work was supported by the Brain Korea 21 Project 2009 and the Korea Research Foundation grant (Grant No. KRF-2008-313-C00258).

${ }^{1}$ A. A. Tseng, A. Notargiacomo, and T. P. Chen, J. Vac. Sci. Technol. B 23, 877 (2005)

${ }^{2}$ W. A. Hofer, A. S. Foster, and A. L. Shluger, Rev. Mod. Phys. 75, 1287 (2003).

${ }^{3}$ J. W. Hong, D. S. Kahng, J. C. Shin, H. J. Kim, and Z. G. Khim, J. Vac. Sci. Technol. B 16, 2942 (1998).

${ }^{4}$ S. V. Kalinin and D. A. Bonnell, Phys. Rev. B 65, 125408 (2002).
${ }^{5}$ S. V. Kalinin and D. A. Bonnell, Nano Lett. 4, 555 (2004).

${ }^{6}$ P. Lehnen, J. Dec, and W. Kleemann, J. Phys. D 33, 1932 (2000).

${ }^{7}$ A. Roelofs, T. Schneller, K. Szot, and R. Waser, Appl. Phys. Lett. 81, 5231 (2002)

${ }^{8}$ J. Y. Son, S. H. Bang, and J. H. Cho, Appl. Phys. Lett. 82, 3505 (2003).

${ }^{9}$ J. Y. Son, G. K. Bog, C. H. Kim, and J. H. Cho, Appl. Phys. Lett. 84, 4971 (2004)

${ }^{10}$ J. Y. Son, K. Kyhm, and J. H. Cho, Appl. Phys. Lett. 89, 092907 (2006)

${ }^{11}$ C. H. Ahn, K. M. Rabe, and J. M. Triscone, Science 303, 488 (2004).

${ }^{12}$ T. Tybell, P. Paruch, T. Giamarchi, and J. M. Triscone, Phys. Rev. Lett. 89, 097601 (2002).

${ }^{13}$ X. Li, A. Mamchik, and I. W. Chen, Appl. Phys. Lett. 79, 809 (2001).

${ }^{14}$ J. Y. Son, C. S. Park, S. K. Kim, and Y. H. Shin, J. Appl. Phys. 104, 064101 (2008).

${ }^{15}$ J. Y. Son and Y.-H. Shin, J. Phys. D 41, 155307 (2008).

${ }^{16}$ P. R. Emtage and J. J. O'Dwyer, Phys. Rev. Lett. 16, 356 (1966).

${ }^{17}$ J. G. Simmons, Phys. Rev. 155, 657 (1967).

${ }^{18}$ Y. S. Yang, S. J. Lee, S. H. Kim, B. G. Chae, and M. S. Jang, J. Appl. Phys. 84, 5005 (1998).

${ }^{19}$ Y. Kim, C. Bae, K. Ryu, H. Ko, Y. K. Kim, S. Hong, and H. Shin, Appl. Phys. Lett. 94, 032907 (2009).

${ }^{20}$ J. F. Scott, Ferroelectric Memories (Springer, New York, 2000).

${ }^{21}$ B. Wang and C. H. Woo, J. Appl. Phys. 94, 4053 (2003).

${ }^{22}$ M. I. Molotskii and M. M. Shvebelman, Philos. Mag. 85, 1637 (2005). 